\title{
Relationship between mental toughness, stress appraisal, and innovation performance of $R \& D$ personnel
}

\author{
Jin Feng \\ Southwest Jiaotong University, Chengdu, Sichuan, China
}

\begin{abstract}
Four hundred and two R\&D personnel were surveyed through questionnaires to study the relationship between mental toughness, stress appraisal (including experience of stress and stress control), and innovation performance. The findings reveal a significant negative correlation between mental toughness and stress experience and a significant positive correlation between mental toughness and both stress control and innovation performance. Furthermore, although the experience of stress was negatively correlated with innovation performance, stress control had the opposite effect. Experience of stress and stress control were the mediating variables for mental toughness and innovation performance, respectively. There was also a significant interaction effect between stress appraisal and mental toughness.
\end{abstract}

Keywords: mental toughness; researchers; stress; innovation performance

\section{INTRODUCTION}

Nowadays, the ability to innovate is an indicator of how competitive an enterprise is. Innovation performance has also become a topic of concern for a long time. This is especially so for the case of R\&D personnel, because their innovation performance is an important factor that contributes towards the innovation performance of enterprises. In today's hi-tech and increasingly competitive world, researchers are facing more stress at work than ever before. While an appropriate amount of stress at work is a good way to motivate staff to be enthusiastic and creative, sustained and excessive work stress often causes job burnout and leads to a detriment in the staff's physical and mental health, which in turn seriously affects their work efficiency.

Mental toughness refers to the quality which determines to a large extent how people deal effectively with challenge, stress, and pressure regardless of prevailing circumstances (Clough \& Strycharczyk, 2012, p.1). It encompasses four aspects: commitment, control, challenge, and confidence (Clough, Earl, \& Sewell, 2002). Commitment refers to the belief in one's purpose and meaning in life; sense of control is the belief that one is able to influence the things that happen around oneself through one's effort; challenge refers to the ability to comprehend negative situations and regard it as a motivating force; and confidence includes having faith in one's abilities and inter-personal skills.

Previous research has shown that individuals with a higher degree of mental toughness were able to handle stress more effectively (Kaiseler, Polman, \& Nicholls, 2009; Nicholls, Polman, Levy, \& Backhouse, 2008) and perform more optimally (Jones, Hanton, \& Connaughton, 2007). Earlier studies relating to stress and innovation performance also generally found that stress was a major factor holding back researchers from achieving their full potential (e.g., Rager, 2005; Bunce, 1996). In view of that, we hypothesized that researchers with strong mental toughness would be better able to manage stress and handle adversity, thereby experiencing less pressure and achieving better innovation performance. The following assumptions were made for this study: (a) mental toughness is negatively correlated with stress experience but positively correlated with stress control and performance innovation; (b) stress experience and stress appraisal are the mediating variables for mental toughness and innovation performance respectively; and (c) there is a significant interaction effect between stress appraisal and mental toughness. The last assumption was made because very few studies had examined this issue and we would like to confirm it. To verify the above-stated assumptions, we examined the relationship between 
the mental toughness and stress appraisal of researchers and their innovation performance.

\section{METHODS}

\subsection{Respondents}

A total of 426 researchers responded to the web-based survey that we conducted. Of the submitted questionnaires, 402 were deemed valid. Of the 402 participants, 195 (48.5\%) were men, and 207 (51.5\%) were women; further, $98(24.4 \%)$ were 30 years and below, 124 (30.8\%) were $31-40$ years, 105 (26.1\%) were $41-49$ years, and $75(18.7 \%)$ were 50 years and above. Participants were employed in universities $(158 ; 39.3 \%)$, research institutes $(113 ; 28.1 \%)$, or corporations and enterprises $(131 ; 32.6 \%)$.

\subsection{Materials}

(1) Mental toughness. This was measured using the Mental Toughness Questionnaire-48 (MTQ48), a questionnaire with a 5-point response scale that was established by Clough et al. (2002). For this study, the internal consistency reliability for the overall scale of the questionnaire and its six dimensions ranged from $0.75-0.87$, indicating a relatively high degree of reliability.

(2) Stress appraisal. Respondents were asked to rate their experience of stress over the past two weeks prior to the survey. A 7-point rating scale was adopted, ranging from 1 (did not feel any stress at all) to 7 (felt extremely stressed). A 7-point rating scale was also used to measure their level of stress control, ranging from 1 (completely out of control) to 7 (completely within control).

(3) Innovation performance. This was measured by using three factors proposed in the study by Brown and Eisenhardt (1995). Respondents were asked to rate, on a 7-point scale, their own standing relative to their peers and competitors in terms of: (a) developing new products or technologies often and regularly, (b) rapidly marketing their new products or technologies, and (c) developing high-quality new products or technologies.

(4) Procedure. A web-based survey was conducted, where respondents were invited to voluntarily complete a questionnaire posted on a designated web site. The collated statistics were analyzed using SPSS 16.0 (SPSS Inc., Chicago, IL).

\section{RESULTS}

Firstly, the scores for mental toughness, experience of stress, stress appraisal, and innovation performance were studied, and the average values and standard deviations derived (see Table 1). It was found that the stress level faced by researchers was generally high $(M=5.096, S D=2.122)$, while their ability to manage stress was relatively low $(M=2.936, S D=2.312)$.

Further analysis showed that stress control $(\mathrm{r}=.326$, $\mathrm{p}<.05)$ and innovation performance $(\mathrm{r}=.496, \mathrm{p}<.01)$ were both positively correlated with mental toughness, while experience of stress was negatively correlated with mental toughness $(\mathrm{r}=-.215, \mathrm{p}<.05)$. However, stress control and innovation performance were both negatively correlated with the experience of stress. Lastly, there was a significant positive correlation between stress control and innovation performance (see Table 1). The above-stated findings verified the

Table 1. Scores for mental toughness, stress appraisal, and innovation performance and relationship between mental toughness, stress appraisal, and innovation performance $(\mathrm{N}=402)$

\begin{tabular}{lllllll}
\hline Subscale & Maximum & Minimum & Mean & Standard Deviation & ES & SC \\
\hline MT & 4.856 & 1.936 & 3.796 & 0.583 & $-.215^{* *}$ & $.326^{* * *}$ \\
ES & 7.000 & 1.000 & 5.096 & 2.122 & $-.517^{* * *}$ & $-.496^{* * *}$ \\
SC & 7.000 & 1.000 & 2.936 & 2.312 & $-612^{* * *}$ \\
IP & 6.748 & 3.854 & 4.869 & .643 & $.397^{* *}$ \\
\hline
\end{tabular}

Note. MT: mental toughness; ES: experience of Stress; SC: stress control; IP: innovation performance. All values are Pearson's correlation coefficients. ( $p<.05, * * p<.01, * * * p<.001)$

Table2. Mediating variables for stress appraisal with mental toughness and innovation performance

\begin{tabular}{llll}
\hline Subscale & Regression Equation & SE & Regression Coefficient \\
\hline Regression for IP with MT & $\mathrm{y}=.434 \mathrm{x}$ & .076 & $\mathrm{t}=6.597^{* * * *}$ \\
Regression for SC with MT & $\mathrm{m}=.425 \mathrm{x}$ & .059 & $\mathrm{t}=5.794^{* * *}$ \\
Regression for IP with MT and SC & $\mathrm{y}=.353 \mathrm{x}$ & .042 & $\mathrm{t}=3.987^{* * *}$ \\
$\mathrm{y}$ & $+.379 \mathrm{~m}$ & .045 & $\mathrm{t}=4.795^{* * *}$ \\
\hline
\end{tabular}

Table3. Mediating variables for the experience of stress with mental toughness and innovation performance

\begin{tabular}{llll}
\hline Subscale & Regression Equation & SE & Regression Coefficient \\
\hline Regression for IP with MT & $\mathrm{y}=.434 \mathrm{x}$ & .076 & $\mathrm{t}=6.597^{* * *}$ \\
Regression for ES with MT & $\mathrm{m}=.549 \mathrm{x}$ & .068 & $\mathrm{t}=7.794^{* * *}$ \\
Regression for IP with MT and ES & $\mathrm{y}=.386 \mathrm{x}$ & .058 & $\mathrm{t}=4.675^{* * *}$ \\
&.$- .419 \mathrm{~m}$ & .062 & $\mathrm{t}=5.869^{* * *}$ \\
\hline
\end{tabular}


assumptions that we had made for the study.

A mediating effect test (Wen, Zhang, Hou, \& Liu, 2004) found that stress control was a partial mediating variable for mental toughness and innovation performance (see Table 2). The experience of stress is another partial mediating variable for mental toughness and innovation performance (see Table 3). Again, the assumptions of this study have been verified.

Using two-factor variable analysis, a significant interaction effect was found between the experience of stress and mental toughness $(F(13,216)=35.629, p$ $<.01)$, as well as between stress control and mental toughness $(F(13,216)=53.918, p<.01)$. Those who scored among the top and bottom $27 \%$ for mental toughness were extracted and categorized into the high and low mental toughness groups, respectively. Analysis of the simple effects of stress experience and stress control for these two groups (Table 4) found no obvious difference between the innovation experience of both groups when stress experience was high $(\geq 5)$. However, as the level of stress experienced reduced, the disparity in the innovation performance between the two groups gradually increased until it reached significance (see Figure 1).

Table 4. Effect of Mental Toughness on the Level of Stress Experience and Stress Appraisal

\begin{tabular}{llllll}
\hline $\begin{array}{l}\text { Experience } \\
\text { of Stress }\end{array}$ & $F$ & $p$ & $\begin{array}{l}\text { Stress } \\
\text { control }\end{array}$ & $F$ & $p$ \\
\cline { 1 - 4 } 1 & 12.034 & .007 & 1 & .038 & .970 \\
2 & 11.159 & .013 & 2 & .363 & .831 \\
3 & 10.264 & .021 & 3 & 1.969 & .415 \\
4 & 7.895 & .034 & 4 & 1.891 & .453 \\
5 & 5.518 & .048 & 5 & 7.653 & .035 \\
6 & 2.032 & .301 & 6 & 8.791 & .027 \\
7 & .609 & .732 & 7 & 10.435 & .015 \\
\hline
\end{tabular}

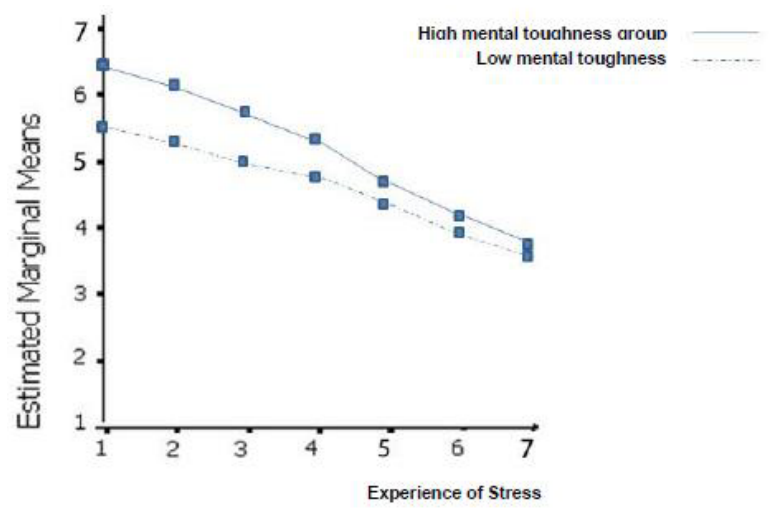

Figure 1. Interaction effect between mental toughness and experience of stress

When stress control was low $(<4)$, there was no obvious difference between the innovation performance of both groups However, the difference between the two groups became increasingly significant as stress control increased, with the high mental toughness group faring much better (see Figure 2).

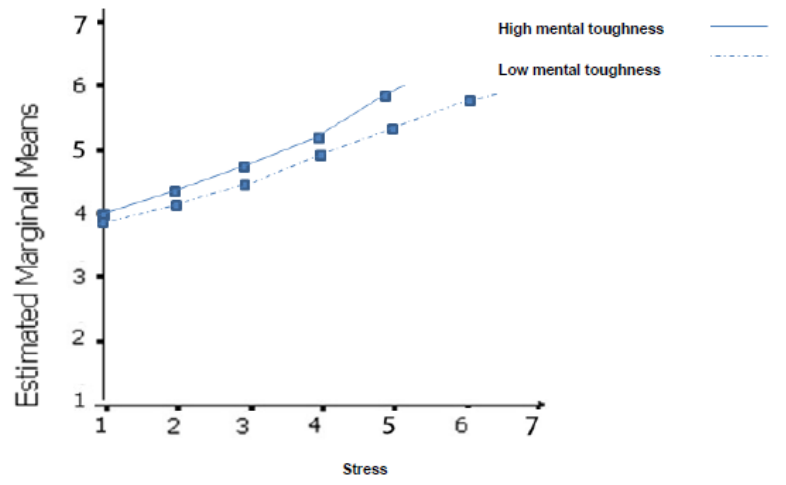

Figure 2. Interaction effect between mental toughness and stress control

\section{DISCUSSION}

The findings of this study show that innovation performance was negatively correlated with the experience of stress, but positively correlated with stress control. Generally, the level of stress experienced by researchers was high, while their stress control was low. Earlier studies have also shown that they experienced high levels of stress, and that there was a negative correlation between stress and innovation performance (Liu et al., 2010). Most researchers have pointed out that it is crucial to have their stress levels reduced, for example, through assignment of a reasonable role, clear definition of job scope, enhanced social support, etc. This study found that mental toughness was positively correlated with stress control, but negatively correlated with the experience of stress. Hence, in addition to the recommendations made by earlier studies, we propose that programs be introduced to assist staff in improving their mental toughness and stress control, while reducing their experience of stress. These measures will lead to better innovation performance. Although this study has established the relationships between mental toughness and both stress control and experience of stress, it did not clarify whether the results were due to high and low mental toughness groups adopting different methods of coping with stress, or the high mental toughness group simply having better coping mechanisms. This issue would need to be studied further in order to establish the theoretical foundation needed to formulate a strategy to increase the mental toughness of staff.

Next, our study found that stress control and the experience of stress were partial mediating variables for mental toughness and innovation performance. Specifically, the stronger one's mental toughness and stress appraisal were, the less pressure one would face, leading to the achievement of higher standards of innovation performance. This finding supports our assumptions on the relationships between the mental toughness and stress appraisal of researchers and their innovation performance. Most of the empirical research on mental toughness was done in relation to the 
ability to cope with stress. For example, the study by Kaiseler et al. (2009) found that mental toughness was negatively correlated with the level of stress experienced by an individual. Other earlier studies on the relationship between mental toughness and innovation performance have also arrived at a similar conclusion - that both were positively correlated (Jones et al., 2007). Using these earlier studies as a foundation, this study went one step further and attempted to clarify the relationship between mental toughness, stress, and innovation performance, while considering stress control as a partial mediating variable for both mental toughness and innovation performance. It should be noted that the experience of stress and stress control were partial - but not complete - mediating variables for mental toughness and innovation performance, respectively. This shows that, in addition to the factor of stress control, mental toughness was able to affect the innovation performance of researchers through other means. Previous studies have established a positive correlation between mental toughness and optimism (Nicholls et al., 2008) and risk-taking (Bull, Shambrook, James, \& Brooks, 2005) etc. The mediating effects of other factors would have to be determined in future studies.

The two-factor variable analysis indicated that mental toughness interacted separately with mental control and experience of stress to affect the innovation performance of staff. On one hand, when the experience of stress was very low or when stress control was very high, the difference in innovation performance between staff in the high and low mental toughness groups was especially apparent. This further illustrates that other mediating variables besides stress control and stress experience were involved in the relationship between mental toughness and innovation performance. On the other hand, when the experience of stress was very high or when stress control was very low, there was no apparent difference in innovation performance between staff in the high and low mental toughness groups. This illustrates that staff members who were mentally tough were able to endure more stress and had better stress control. However, when the level of stress exceeded manageable levels, mental toughness had little impact on innovation performance. This finding further illustrates the need to reduce stress faced by staff, even as their mental toughness is being enhanced. However, it should be noted that this study only observed the overall experience of stress faced by staff and their stress control and did not study the relationship between different types of stress and the overall experience of stress and stress control. As such, we are not able to claim whe- ther it is necessary to consider the different types of stress when reducing stress faced by staff, nor how the different types of stress can be separately managed. Future research into these areas will be needed in order to arrive at a theoretical basis for the reasonable reduction of stress.

\section{CONCLUSION}

There was a significant negative correlation between mental toughness and stress experience and significant positive correlations between mental toughness and both stress control and innovation performance. Furthermore, the experience of stress was negatively correlated with innovation performance, but stress control had the opposite effect. Experience of stress and stress control were the mediating variables for mental toughness and innovation performance respectively. There was also a significant interaction effect between stress appraisal and mental toughness.

\section{REFERENCES}

[1] Brown, S.L. \& Eisenhardt, K.M. 1995. Product development: Past research, present findings, and future directions. Academy of Management Review, 20: 343-378.

[2] Bull, S.J., Shambrook, C.J., James, W. \& Brooks, J.E. 2005. Towards an understanding of mental toughness in elite English cricketers. Journal of Applied Sport Psychology, 17: 209-227.

[3] Bunce, D. 1996. Stress management and innovation interventions at work. Human Relations, 49: 209-232.

[4] Clough, P.J., Earle, K. \& Sewell, D. 2002. Mental toughness: The concept and its measurement. In I. Cockerill (Ed.), Solutions in Sport Psychology, London: Thomson Publishing, pp.32-43.

[5] Clough, P. \& Strycharczyk, D. 2012. Developing Mental Toughness. London: Kogan.

[6] Jones, G., Hanton, S. \& Connaughton, D. 2007. A framework of mental toughness in the world's best performers. The Sport Psychologist, 21: 243-264.

[7] Kaiseler, M., Polman, R. \& Nicholls, A. 2009. Mental toughness, stress, stress appraisal, coping and coping effectiveness in sport. Personality and Individual Differences, 47: 728-733.

[8] Nicholls, A. R., Polman, R. C., Levy, A. R. \& Backhouse, S. 2008. Mental toughness, optimism, pessimism, and coping among athletes. Personality and Individual Differences, 44: 1182-1192.

[9] Rager, K. 2005. Self-care and the qualitative researcher: When collecting data can break your heart. Educational Researcher, 34: 23-27.

[10] Wen, Z.L., Zhang, L., Hou, T.J. \& Liu, H.Y. 2004. Testing and application of the mediating effects (in Chinese). Acta Psychologica Sinica, 35: 802-809. 